\title{
Reliability and reproducibility of measurements in para-sagittal planes on sub-axial cervical vertebral bodies: a morphometric study of endplates in three- dimensional models
}

\author{
Long Wang ${ }^{1}$, Hao T. Luo ${ }^{2,3}$, Wei Lư ${ }^{2}$, Xing Bo Cai ${ }^{1}$, Chen Yu ${ }^{1}$ and Sheng Lu ${ }^{2,3^{*}}$ (D)
}

\begin{abstract}
Background: Dimensional measurements have been implemented on a variety of entities in morphological studies of the sub-axial cervical vertebral endplate. Despite great progress, little information between the mid-sagittal plane and bilateral uncinate processes has been acquired due to the lack of a reliable method to determine the parasagittal planes. Also, few studies of this region are available. We proposed a new approach to defining the parasagittal planes on a 3D cervical vertebral body model; in this approach, dimensions can be measured in a specific plane. The aim of this study was to assess the inter-observer and intra-observer reliability of the measurements in different sagittal planes on sub-axial cervical vertebral endplates of 3D models.

Methods: We established mid-sagittal and bilateral quarter para-sagittal planes on the 3D model of a sub-axial cervical vertebral body based on landmarks labeled on the surface. By intersecting the vertebral body with the planes, three curves located at the three para-sagittal planes were generated. Linear dimensions were measured on every curve by two observers separately, and in total, 24 sub-axial cervical spines were included in the study. The first observer (O1) performed the procedure twice with an interval of 2 weeks. The paired $t$ test, Wilcoxon matchedpairs signed-rank test and the interclass correlation coefficient (ICC) were employed to evaluate the inter- and intraobserver reliability of the proposed method.

Results: There were no significant differences in most intra- and inter-observer comparisons, and higher nonsignificant proportions were found in the intra-observer comparisons than in comparisons between different observers. The interclass correlation coefficients (ICCS) in the measurements were excellent (>0.75) in most circumstances, and the values in intra-observer comparisons were higher than those in inter-observer comparisons.
\end{abstract}

\footnotetext{
* Correspondence: lushengar@163.com

2Department of Orthopaedics, The First People's Hospital of Yunnan

Province, No.157, Jinbi Rd, Kunming, Yunnan, China

${ }^{3}$ Key Laboratory of Digital Orthopedics of Yunnan Province, No.157, Jinbi Rd, Kunming, Yunnan, China

Full list of author information is available at the end of the article
}

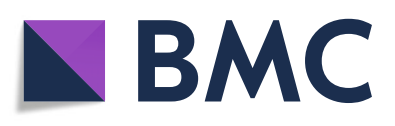

(- The Author(s). 2021 Open Access This article is licensed under a Creative Commons Attribution 4.0 International License, which permits use, sharing, adaptation, distribution and reproduction in any medium or format, as long as you give appropriate credit to the original author(s) and the source, provide a link to the Creative Commons licence, and indicate if changes were made. The images or other third party material in this article are included in the article's Creative Commons licence, unless indicated otherwise in a credit line to the material. If material is not included in the article's Creative Commons licence and your intended use is not permitted by statutory regulation or exceeds the permitted use, you will need to obtain permission directly from the copyright holder. To view a copy of this licence, visit http://creativecommons.org/licenses/by/4.0/ The Creative Commons Public Domain Dedication waiver (http://creativecommons.org/publicdomain/zero/1.0/) applies to the data made available in this article, unless otherwise stated in a credit line to the data. 
Conclusions: In this study, we proposed an approach to determine the bilateral quarter para-sagittal planes in a 3D cervical vertebral body model; the results demonstrated that the method is reproducible with high intra- and interobserver agreement.

Keywords: Cervical endplate morphology, 3D model, Para-sagittal planes, Dimension measurement

\section{Background}

Morphometric studies of sub-axial cervical endplates have increased over the past decades [1], and the main purpose is to provide anatomic data for the design or optimization of the intervertebral instrument and preoperative planning. Dimensional measurements of the endplates have been carried out over a series of entities, from cadaveric vertebrae and radiographic images in the early days of implementing this approach $[2,3]$ and have progressed to include technically advanced methods such as CT and MRI in recent years $[4,5]$. Also, as a newly developed technique in morphological studies, digitized three-dimensional (3D) models have become more and more popular since their emergence [5-8]. Among the dimensions measured in these studies, depth and width are the most frequently implemented, angular dimensions were common in many studies, and the radius or diameter of an arc could be easily calculated by fitting a curve with the assistance of computer programs [9].

Although significant progress has been made in medical imaging, image segmentation, 3D model generation, and measuring techniques, further geometric knowledge remains to be elucidated at the region between the midsagittal plane (MSP) and bilateral uncinate processes (UPs), and a limited number of studies are available. One possible explanation is that it is not easy to accurately determine these regions on cadaveric vertebral bodies due to the few identifiable anatomic landmarks available. Panjabi et al. made para-sagittal sections of cadaveric vertebral bodies in increments of $5 \mathrm{~mm}$ and used a cutting machine to measure the thickness of the cortical bone of cervical vertebral bodies [10]. In another study by Ebraheim et al., bilateral para-sagittal planes were defined on a cervical vertebra $5 \mathrm{~mm}$ lateral to the mid-sagittal plane to evaluate the safety of anterior screw placement [11]. Feng et al. defined the curves on parasagittal planes in a 3D model generated from optical scans of a cadaveric vertebral endplate [5] in a recent study.

In this study, we proposed an approach to define the bilateral quarter para-sagittal planes on a 3D sub-axial cervical vertebra model based on landmarks labelled on the anatomic features of the vertebral bodies; measurements can be carried out on these planes. The series of dimensions can better represent the shape of the endplates. The objective of the study was to assess the reliability and reproducibility of the method between two different observers.

\section{Method \\ Data collection}

Twenty-four CT scans of the sub-axial cervical spine were included in this study after we retrospectively reviewed the database of the hospital. All CT data were collected under the same scanning standard (slice thickness $0.625 \mathrm{~mm}$, LightSpeed VCT, GE medical system, USA). Evaluated patients included 12 males, with a mean age of $34.5 \pm 14.8$ years (18-61 years), and 12 females, aged $40.2 \pm 14.7$ years (18-63 years). All CT data were selected by two senior residents specializing in spine surgery and subsequently reviewed by one specialist in the same subject. The inclusion criteria mainly included images of the adult cervical spine. Participants with pathological conditions of the cervical spine, such as severe degeneration, deformities, tumor, trauma, and previous surgeries were excluded. All participants in the study were informed of the research and approved the use of their data in this study. All patients from whom CT data were acquired were anonymized. The institutional board of review approved this study.

\section{Segmentation and 3D model reconstruction}

The CT data were segmented based on the CT value (> $226 \mathrm{HU}$ ), and a 3D surface model of every sub-axial vertebra was generated (Mimics 19.0, Materialise, Leuven, Belgium) (Fig. 1).

\section{Reference plane establishment and curve generation}

After importing the 3D surface model into the engineering software 3-Matics 11.0 (Materialise, Leuven, Belgium), we generated the mid-sagittal plane (MSP) of the vertebral body through landmarks labelled on the vertebral body and the intersection of the bilateral laminae (Fig. 2). After that, two lateral para-sagittal planes were built through the landmarks positioned on the tips of the uncinate processes (UPs) of both sides (Fig. 2). Last, two quarter para-sagittal planes (QPSPs) of the vertebral body were calculated as the average planes between the mid-sagittal plane and the lateral planes (Fig. 2).

By intersecting the vertebral body with the three planes, the mid-sagittal plane and two quarter para- 

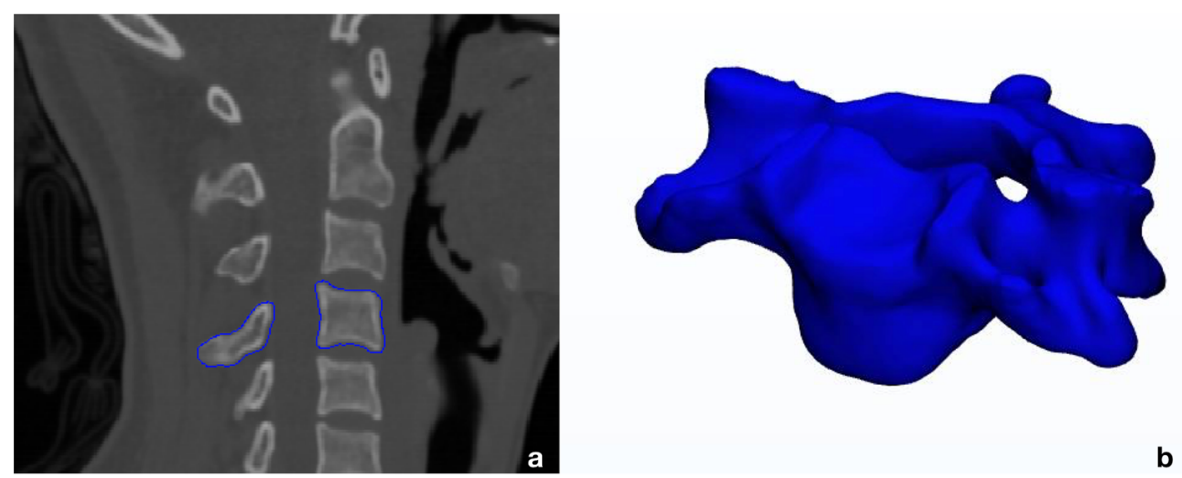

b

Fig. 1 Segmentation of the C4 vertebra (a) and the 3D surface model generated (b)

sagittal planes, three closed curves were generated (Fig. 2).

\section{Measurement}

We then exported the three curves into the 3D modelling software Rhinoceros 6.0 (Rhinoceros, Robert McNeel and Associates, Seattle, Washington, USA) for further assessment. After re-alignment in the coordinate system, the three curves were evaluated separately (Fig. 3). Three linear dimensions on every single curve were obtained, including the anteroposterior depth of the superior (SED) and the inferior endplate (IED), according to the manner proposed by Panjabi et al. [10]; the depth of inferior endplate concave (IECD) (Fig. 3); and the distance between anterior and posterior rims of the inferior endplate concave [12].

Two observers, including one musculoskeletal digitization engineer (observer one, O1) and one spine surgeon in fellowship training (observer two, O2), both of whom were experienced in 3D modelling software and were familiar with the procedures. The two observers independently performed modelling assessments and measurements of all enrolled sub-axial cervical vertebrae (C3-C7), and one of them (observer one, O1) repeated the modelling and measurements twice with an assessment interval of two weeks.

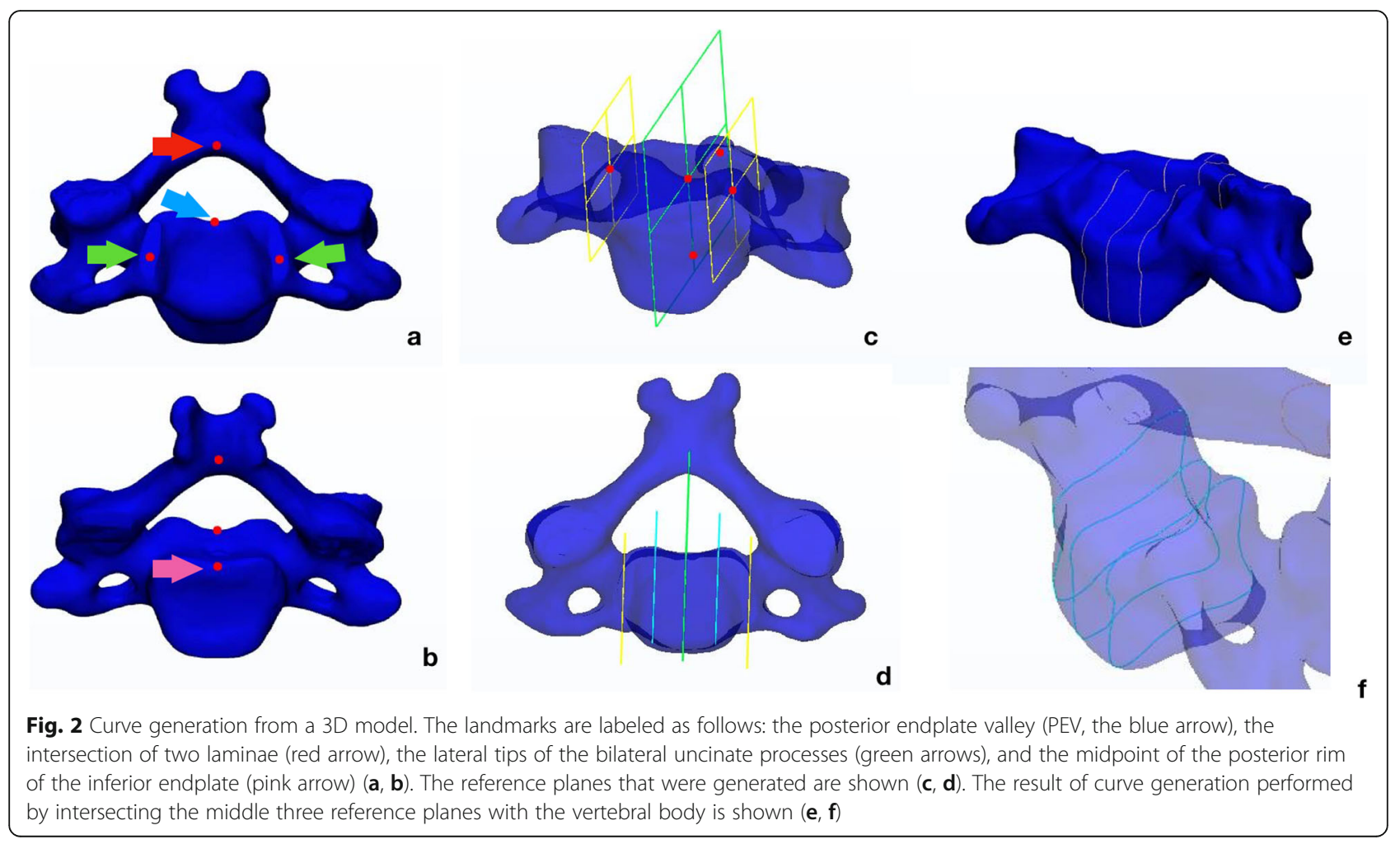



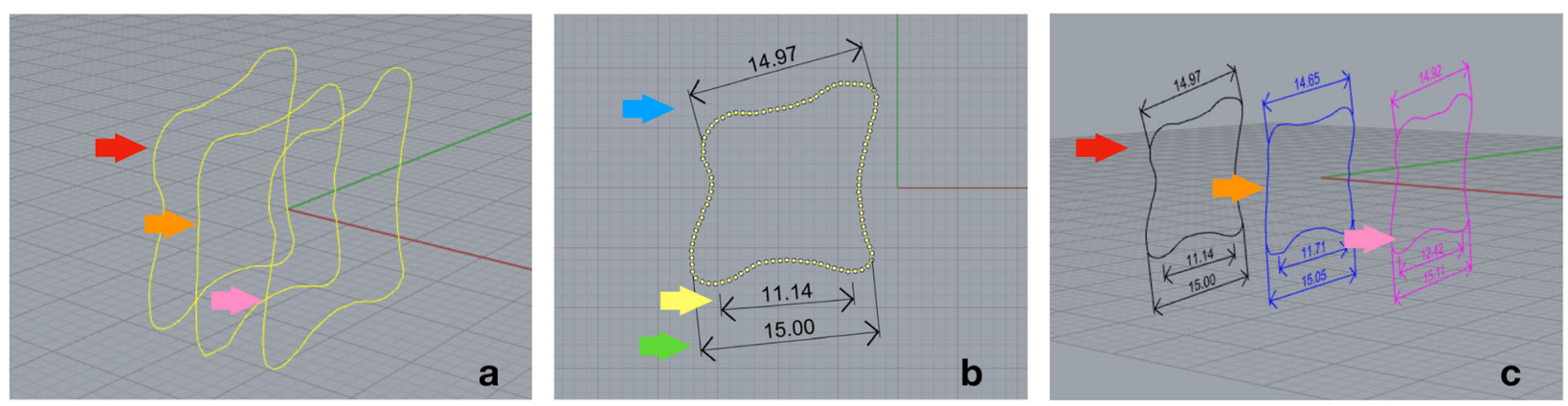

Fig. 3 Measurements in the three planes. Re-alignment in the coordinate system was performed in the following order: right quarter para-sagittal plane (red arrow), mid-sagittal plane, and left quarter para-sagittal plane (a). Measurements of the curve on the right quarter para-sagittal plane, depth of the superior endplate (RSED, blue arrow), depth of the inferior endplate concave (RIECD, yellow arrow), depth of the inferior depth (RIED, green arrow) (b). Spatial display of the three sagittal planes with measurements (c)

\section{Statistical analysis}

The statistical software packages SPSS 18.0 (SPSS Inc., Chicago, IL, USA) and Prism 8.0 (GraphPad, USA) were employed to analyze the data and draw the graphs. Descriptive statistics was used for general data analysis, and the paired $t$ test and the Wilcoxon matched-pairs signed-rank test were used for the comparison of the measurements. Interclass correlation coefficients (ICCs) were applied for the inter-observer and intra-observer reliability evaluation; $p<0.05$ was considered to indicate statistical significance.

\section{Result}

The values measured in males were greater than those in females and increased from $\mathrm{C} 3$ to $\mathrm{C} 7$ in almost all dimensions, (Fig. 4), and the trends of variations between men and women were similar. The discrepancies between the depth of the superior endplate (SED) and depth of the inferior endplate (IED) declined from C3 to C7 in the three sagittal planes (Fig. 4). The values measured on mid-sagittal planes and trends were consistent with the other studies of the measurement of the subaxial cervical vertebra $[1,4,7]$. There were no significant differences between the values measured by the two observers and observer one (O1).

For the comparisons between values measured by the two independent observers and measured by $\mathrm{O} 1$ at different times, a paired $t$ test was employed, and for measurement values that did not fit the normality distribution (Shapiro-Wilk test), Wilcoxon matchedpairs signed-rank test was employed. No significant differences existed in most comparisons, and there were higher non-significant proportions in the intra-observer comparisons compared with the comparisons between different observers (Tables 1 and 2).

Inter-class coefficients (ICC) were used for the analysis of reproducibility between the two observers and between the measurements taken at different times by $\mathrm{O} 1$.
The results indicated that most ICC values were greater than 0.75 . The ICCs were higher in the intra-observer comparisons (0.777-0.998 in females, $0.565-0.993$ in males) than the inter-observer (0.602-0.965 in females, $0.403-0.958$ in males) comparisons (Tables 3 and 4; Fig. 5).

\section{Discussion}

\section{The result}

The results indicated that the interclass correlation coefficients (ICCs) were excellent (> 0.75) for most occasions, and the inter-observer and intra-observer results were reliable and reproducible.

The main reason for such a result can be attributed to the fact that most landmarks we chose to establish sagittal planes had already been verified in pre-existing studies; these landmarks include the posterior endplate valley (PEV), which is a newly proposed position for landmark labeling [13] and the intersection of the bilateral laminae [14]. Both of these landmarks are stable anatomic structures as their stability guarantees the stability of the mid-sagittal plane establishment. Moreover, the midpoint of the posterior rim of the inferior endplate can be identified as a candidate landmark; the uncinate processes are stable anatomical structures located at the lateral borders of the vertebral bodies [7, 15-17]. Therefore, the mid-sagittal plane and the two following quarter para-sagittal planes can be accurately determined and established, with high reliability between observers with different occupational backgrounds as well as under different circumstances.

There are also other methods to determine the midsagittal plane on CT images and 3D models. The largest spinous in the sagittal plane was referred to as the midsagittal plane in CT data-based studies [4, 12]; for 3D models, mid-points of three lines created by connecting bilateral anatomical structures were used to generate the mid-sagittal plane [18]. 


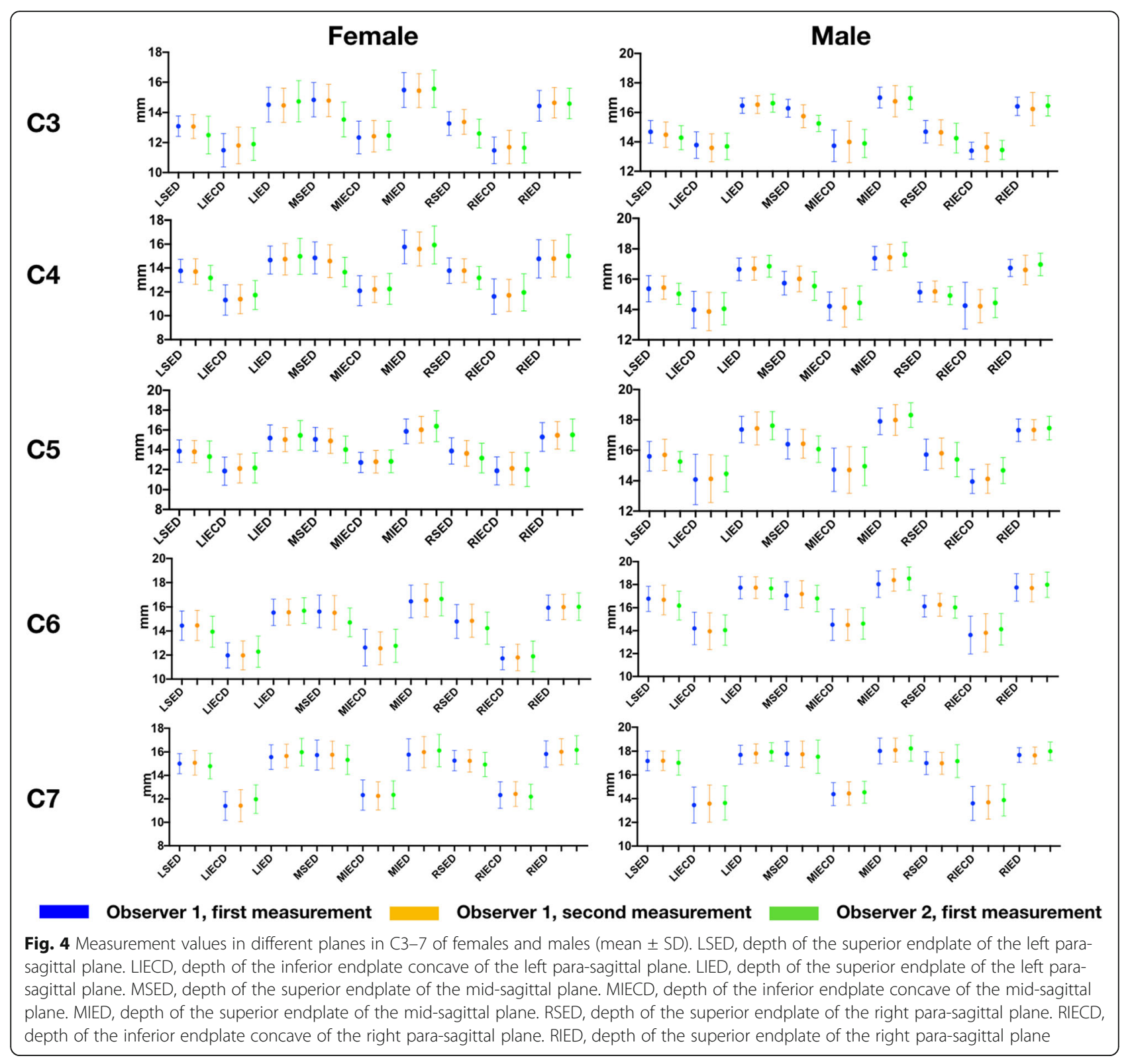

\section{Measurement modalities}

Over a long period, the most widely used and accepted method for cervical vertebrae measurement is direct measurement over the dry bone with a calliper [11, 19]. Radiographic data have been proposed, for instance [2]; however, due to the inherent disadvantages of these data, errors can be easily generated from magnification, rotation and projection, and direct measurement remains the mainstay $[20,21]$. For many researchers, human bone is the preferred option rather than the ideal option, mainly due to the difficulties in obtaining and carrying bone; also, the reproducibility of the measurements in certain regions is also in doubt, especially for regions with few identifiable anatomic landmarks. The emergence of modern medical imaging modalities, such as CT and MRI, brought new considerations into endplate measurements; also, high-resolution image data have many advantages, especially with the assistance of computer programs. Gradually, CT and MRI imagebased measurements have replaced the predominant position in the first decade of the twenty-first century.

Included but not limited to measurement, a digital 3D bone model, generated from CT, MRI images, and optical scanning, can serve as an alternative to replace cadaveric bone; many shortcomings of dry bone can be overcome thanks to modelling and engineering 
Table 1 Comparison between different measurements in female

\begin{tabular}{|c|c|c|c|c|c|c|c|c|c|c|c|c|c|c|c|c|c|c|}
\hline & \multicolumn{2}{|l|}{ LSED } & \multicolumn{2}{|c|}{ LIECD } & \multicolumn{2}{|l|}{ LIED } & \multicolumn{2}{|c|}{ MSED } & \multicolumn{2}{|c|}{ MIECD } & \multicolumn{2}{|l|}{ MIED } & \multicolumn{2}{|l|}{ RSED } & \multicolumn{2}{|c|}{ RIECD } & \multicolumn{2}{|l|}{ RIED } \\
\hline & Intra & Inter & Intra & Inter & Intra & Inter & Intra & Inter & Intra & Inter & Intra & Inter & Intra & Inter & Intra & Inter & Intra & Inter \\
\hline C3 & 0.72 & 0.03 & 0.05 & 0.02 & $0.45^{*}$ & 0.18 & 0.30 & $<0.01$ & 0.24 & 0.10 & 0.39 & 0.40 & 0.08 & $<0.01$ & 0.13 & 0.28 & $<0.01^{*}$ & $0.29^{*}$ \\
\hline C4 & 0.45 & 0.01 & 0.64 & $<0.01$ & 0.74 & 0.13 & 0.01 & $<0.01$ & 0.23 & 0.17 & 0.03 & 0.27 & 0.98 & $<0.01$ & 0.43 & 0.1 & 0.75 & 0.17 \\
\hline C5 & 0.63 & 0.04 & 0.13 & 0.09 & 0.59 & 0.08 & 0.10 & $<0.01$ & 0.55 & 0.54 & 0.40 & 0.06 & 0.42 & $<0.01$ & $0.15^{*}$ & 0.60 & 0.02 & 0.20 \\
\hline C6 & 0.79 & $<0.01$ & 0.97 & 0.17 & 0.41 & 0.16 & 0.28 & $<0.01$ & 0.71 & 0.34 & 0.50 & 0.10 & 0.33 & $<0.01$ & 0.31 & 0.40 & 0.37 & 0.50 \\
\hline C7 & 0.37 & 0.12 & 0.87 & 0.01 & 0.06 & 0.02 & $0.65^{*}$ & 0.02 & 0.53 & 0.92 & 0.06 & 0.08 & 0.77 & 0.02 & 0.12 & 0.40 & 0.06 & 0.03 \\
\hline
\end{tabular}

*Wilcoxon matched-pairs signed-rank test

LSED depth of the superior endplate of the left para-sagittal plane, LIECD depth of the inferior endplate concave of the left para-sagittal plane, LIED depth of the superior endplate of the left para-sagittal plane, MSED depth of the superior endplate of the mid-sagittal plane, MIECD depth of the inferior endplate concave of the mid-sagittal plane, MIED depth of the superior endplate of the mid-sagittal plane, RSED depth of the superior endplate of the right para-sagittal plane, RIECD depth of the inferior endplate concave of the right para-sagittal plane, RIED depth of the superior endplate of the right para-sagittal plane

Table 2 Comparison between different measurements in male

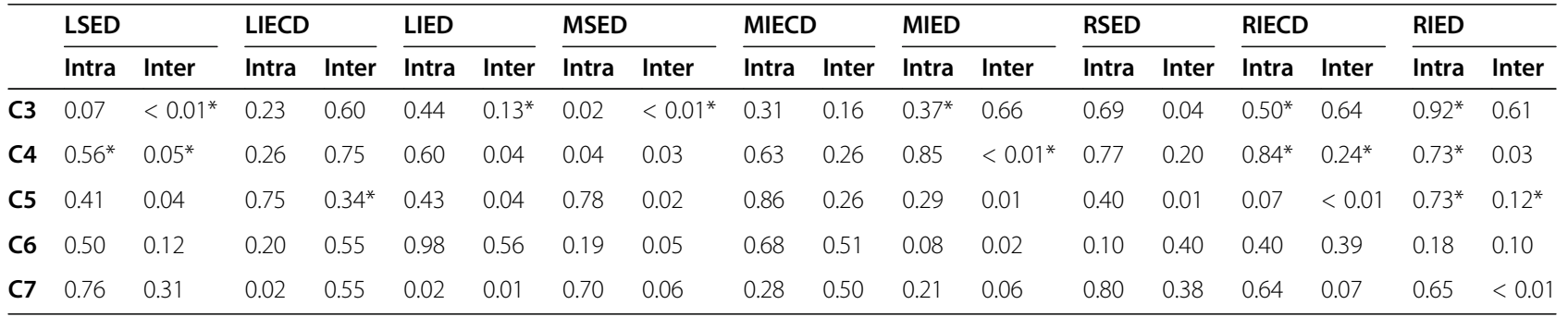

* Wilcoxon matched-pairs signed-rank test

LSED depth of the superior endplate of the left para-sagittal plane, LIECD depth of the inferior endplate concave of the left para-sagittal plane, LIED depth of the superior endplate of the left para-sagittal plane, MSED depth of the superior endplate of the mid-sagittal plane, MIECD depth of the inferior endplate concave of the mid-sagittal plane, MIED depth of the superior endplate of the mid-sagittal plane, RSED depth of the superior endplate of the right para-sagittal plane, RIECD depth of the inferior endplate concave of the right para-sagittal plane, RIED depth of the superior endplate of the right para-sagittal plane

Table 3 Inter-rater and intra-rater interclass correlation coefficient(ICC) in female group

\begin{tabular}{|c|c|c|c|c|c|c|c|c|c|c|c|c|c|c|c|c|c|c|}
\hline & \multicolumn{2}{|l|}{ LSED } & \multicolumn{2}{|c|}{ LIECD } & \multicolumn{2}{|l|}{ LIED } & \multicolumn{2}{|l|}{ MSED } & \multicolumn{2}{|c|}{ MIECD } & \multicolumn{2}{|l|}{ MIED } & \multicolumn{2}{|l|}{ RSED } & \multicolumn{2}{|l|}{ RIECD } & \multicolumn{2}{|l|}{ RIED } \\
\hline & Intra & Inter & Intra & Inter & Intra & Inter & Intra & Inter & Intra & Inter & Intra & Inter & Intra & Inter & Intra & Inter & Intra & Inter \\
\hline C3 & 0.965 & 0.602 & 0.888 & 0.839 & 0.970 & 0.899 & 0.990 & 0.732 & 0.975 & 0.965 & 0.990 & 0.965 & 0.969 & 0.690 & 0.877 & 0.841 & 0.960 & 0.934 \\
\hline C4 & 0.996 & 0.765 & 0.917 & 0.920 & 0.777 & 0.870 & 0.959 & 0.673 & 0.988 & 0.955 & 0.979 & 0.948 & 0.956 & 0.751 & 0.977 & 0.895 & 0.998 & 0.934 \\
\hline$C 5$ & 0.974 & 0.798 & 0.917 & 0.903 & 0.781 & 0.947 & 0.959 & 0.698 & 0.950 & 0.868 & 0.888 & 0.776 & 0.817 & 0.833 & 0.929 & 0.869 & 0.992 & 0.932 \\
\hline C6 & 0.995 & 0.839 & 0.883 & 0.778 & 0.997 & 0.937 & 0.976 & 0.698 & 0.929 & 0.943 & 0.962 & 0.952 & 0.992 & 0.847 & 0.979 & 0.829 & 0.996 & 0.935 \\
\hline C7 & 0.972 & 0.833 & 0.945 & 0.773 & 0.985 & 0.838 & 0.975 & 0.873 & 0.961 & 0.875 & 0.961 & 0.874 & 0.961 & 0.898 & 0.992 & 0.877 & 0.952 & 0.880 \\
\hline
\end{tabular}

LSED depth of the superior endplate of the left para-sagittal plane, LIECD depth of the inferior endplate concave of the left para-sagittal plane, LIED depth of the superior endplate of the left para-sagittal plane, MSED depth of the superior endplate of the mid-sagittal plane, MIECD depth of the inferior endplate concave of the mid-sagittal plane, MIED depth of the superior endplate of the mid-sagittal plane, RSED depth of the superior endplate of the right para-sagittal plane, RIECD depth of the inferior endplate concave of the right para-sagittal plane, RIED depth of the superior endplate of the right para-sagittal plane 
Table 4 Inter-rater and intra-rater interclass correlation coefficient(ICC) in female group

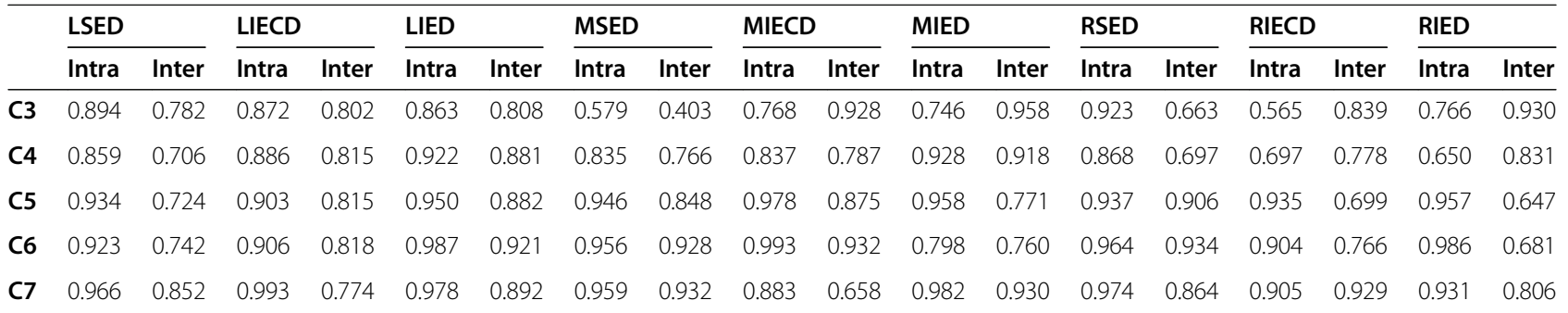

LSED depth of superior endplate of left para-sagittal plane, LIECD depth of inferior endplate concave of left para-sagittal plane, LIED depth of superior endplate of left para-sagittal plane, MSED depth of superior endplate of mid-sagittal plane, MIECD depth of inferior endplate concave of mid-sagittal plane, MIED depth of superior endplate of mid-sagittal plane, RSED depth of superior endplate of right para-sagittal plane, RIECD depth of inferior endplate concave of right parasagittal plane, RIED depth of superior endplate of right para-sagittal plane

computer programs. Many in silico-based studies on 3D models have been implemented $[5,6,8,18]$ by the development of software, and studies on 3D models will increase in the future.

The 3D model has advantages, such as being relatively easier to obtain, being capable of repeated use, having fewer ethics burdens, being able to register with other image modalities, being applicable to computational simulations, and providing different authors the ability to verify the accuracy of the $3 \mathrm{D}$ model.

\section{Future prospects}

The method we used in this study has the potential to be expanded into a wider area of applications. Different portions of an object can be accurately positioned at the coronal, sagittal, and axial planes for further evaluation. For example, by incorporating reference planes, measurements on cadaveric bones can also be augmented in the case of augmented reality (AR) equipment and other similar devices. Furthermore, more detailed landmarks can be generated by intersecting curves on different planes for modelling in advanced computational anatomical research.

\section{Limitation of the study: need further exploration}

One limitation of the study is that the method has only been verified on vertebrae with no or mild degeneration, and the landmarks may be difficult to recognize in cases of severe degeneration; thus, further exploration is needed. In this study, the shape of the vertebral body is regular and has a relatively simpler topology; however, when implementing similar studies in more complex bone, such as the pelvis, or for bones in which it is difficult to define a datum reference plane, such as the humerus and femur, the method may need further adaption. Another limitation is that the samples enrolled in the study were relatively smaller, observers were limited to experienced digital medicine practitioners, and questions remain concerning further expansion to a larger population and to include observers. Additionally, measurements were carried out only on the sagittal plane, and more modifications may be considered when applied to coronal and axial planes.

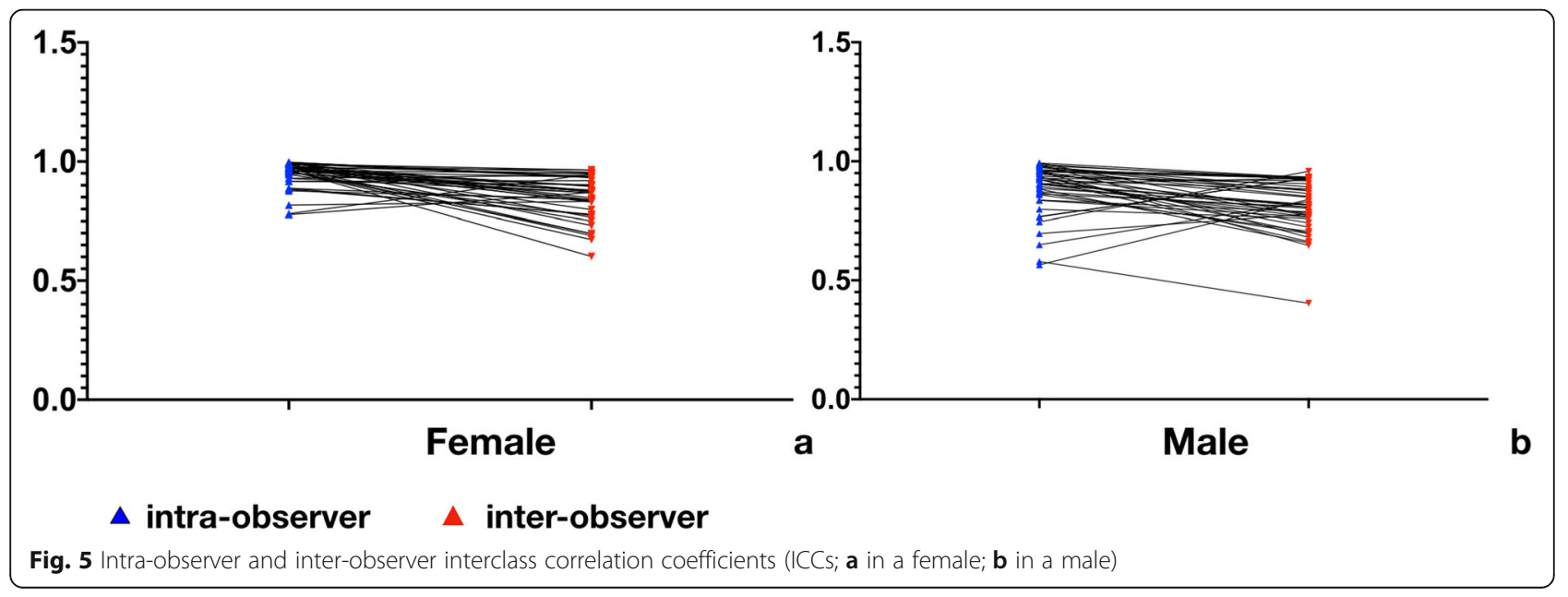




\section{Conclusion}

In this study, we propose an approach for determining the bilateral quarter para-sagittal planes on a $3 \mathrm{D}$ cervical vertebral body model; the reproducibility in a single observer and intra-observer reproducibility was evaluated, and the results demonstrated that the method is reproducible with high intra- and inter-observer agreement.

\section{Abbreviations}

3D: Three dimensional; CT: Computed tomography; MRI: Magnetic resonance imaging; HU: Houndsfield unit; UP: Uncinate process; ICC: Interclass correlation coefficient; PEV: Posterior endplate valley; SED: Superior endplate; IED: Inferior endplate; QPSP: Quarter para-sagittal planes; LSED: Depth of superior endplate of left para-sagittal plane; LIECD: Depth of inferior endplate concave of left para-sagittal plane; LIED: Depth of superior endplate of left para-sagittal plane; MSED: Depth of superior endplate of mid-sagittal plane; MIECD: Depth of inferior endplate concave of mid-sagittal plane; MIED: Depth of superior endplate of mid-sagittal plane; RSED: Depth of superior endplate of right para-sagittal plane; RIECD: Depth of inferior endplate concave of right para-sagittal plane; RIED: Depth of superior endplate of right para-sagittal plane; AR: Augmented reality

\section{Acknowledgements}

The authors would like to thank Doctor Wu Ying for the inspiration she gave us.

\section{Authors' contributions}

WL wrote the manuscript text and finished the statistical analysis. LHT and LW performed the measurements. CXB and YC picked the data. LS is the corresponding author and made the planning of the study. All authors read and approved the final manuscript.

\section{Funding}

Joint special fund of Applied Fundamental Research of Kunming Medical University granted by Science and Technology Office of Yunnan, grant no. 202001AY070001-172.

\section{Availability of data and materials}

All data generated or analyzed during this study are included in this published article.

\section{Declarations}

\section{Ethics approval and consent to participate}

This study, involving human CT data, has been performed in accordance with the Declaration of Helsinki, and the ethics committee of the First People's Hospital of Yunnan Province approved the study (Serial No.KHLL2019-KY033). All participants have been informed of the study and approved of using their CT data. Informed consent was obtained from all individual participants included in the study.

\section{Consent for publication}

Not applicable.

\section{Competing interests}

The authors declare that they have no competing interests.

\section{Author details}

${ }^{1}$ Postgraduate College of Kunming Medical University, No.1168 Chunrong Xi Road, Chenggong District, Kunming, China. 'Department of Orthopaedics, The First People's Hospital of Yunnan Province, No.157, Jinbi Rd, Kunming, Yunnan, China. ${ }^{3}$ Key Laboratory of Digital Orthopedics of Yunnan Province, No.157, Jinbi Rd, Kunming, Yunnan, China.
Received: 3 May 2021 Accepted: 2 August 2021

Published online: 16 August 2021

\section{References}

1. Feng H, Fang XY, Huang DG, Yu CC, Li HK, Zhao SC, et al. A morphometric study of the middle and lower cervical vertebral endplates and their components. Medicine (Baltimore). 2017;96(10):e6296. https://doi.org/10.1 097/md.0000000000006296.

2. Katz PR, Reynolds HM, Foust DR, Baum JK. Mid-sagittal dimensions of cervical vertebral bodies. American journal of physical anthropology. 1975; 43(3):319-26. https://doi.org/10.1002/ajpa.1330430304.

3. Kokubun S, Sakurai M, Tanaka Y. Cartilaginous endplate in cervical disc herniation. Spine. 1996;21(2):190-5. https://doi.org/10.1097/00007632-1 99601150-00006.

4. Lou J, Liu H, Rong X, Li H, Wang B, Gong Q. Geometry of inferior endplates of the cervical spine. Clinical neurology and neurosurgery. 2016;142:132-6. https://doi.org/10.1016/j.clineuro.2016.01.027.

5. Feng H, Li H, Ba Z, Chen Z, Li X, Wu D. Morphometry evaluations of cervical osseous endplates based on three dimensional reconstructions. International orthopaedics. 2019;43(6):1521-8. https://doi.org/10.1007/s002 64-018-4053-1.

6. Kim MK, Kwak DS, Park CK, Park SH, Oh SM, Lee SW, et al. Quantitative anatomy of the endplate of the middle and lower cervical vertebrae in Koreans. Spine. 2007:32(14):E376-81. https://doi.org/10.1097/BRS.0b013e31 8067 384.

7. Zhu YH, Cheng KL, Zhong Z, Li YQ, Zhu QS. Morphologic evaluation of Chinese cervical endplate and uncinate process by three-dimensional computed tomography reconstructions for helping design cervical disc prosthesis. Journal of the Chinese Medical Association : JCMA. 2016;79(9): 500-6. https://doi.org/10.1016/j.jcma.2016.04.003.

8. Yao Q, Yin P, Khan K, Tsai TY, Li JS, Hai Y, et al. Differences of the Morphology of Subaxial Cervical Spine Endplates between Chinese and White Men and Women. BioMed research international. 2018;2018:28541758. https://doi.org/10.1155/2018/2854175.

9. Zhao S, Hao D, Jiang Y, Huang D, Ge C, Feng H. Morphological studies of cartilage endplates in subaxial cervical region. European spine journal : official publication of the European Spine Society, the European Spinal Deformity Society, and the European Section of the Cervical Spine Research Society. 2016;25(7):2218-22. https://doi.org/10.1007/s00586-015-4336-9.

10. Panjabi MM, Chen NC, Shin EK, Wang JL. The cortical shell architecture of human cervical vertebral bodies. Spine. 2001;26(22):2478-84. https://doi. org/10.1097/00007632-200111150-00016.

11. Ebraheim NA, Fow J, Xu R, Yeasting RA. The vertebral body depths of the cervical spine and its relation to anterior plate-screw fixation. Spine. 1998; 23(21):2299-302. https://doi.org/10.1097/00007632-199811010-00010.

12. Chen H, Zhong J, Tan J, Wu D, Jiang D. Sagittal geometry of the middle and lower cervical endplates. European spine journal : official publication of the European Spine Society, the European Spinal Deformity Society, and the European Section of the Cervical Spine Research Society. 2013;22(7):1570-5. https://doi.org/10.1007/s00586-013-2791-8.

13. Aleem IS, Alder J, Popper J, Freedman B, Nassr A, Bydon M, et al. A Novel Anatomic Landmark to Assess Adequate Decompression in Anterior Cervical Spine Surgery: The Posterior Endplate Valley (PEV). Clinical spine surgery. 2019;32(8):345-9. https://doi.org/10.1097/bsd.0000000000000877.

14. Boyle JJ, Singer KP, Milne N. Morphological survey of the cervicothoracic junctional region. Spine. 1996;21(5):544-8. https://doi.org/10.1097/ 00007632-199603010-00003.

15. Tubbs RS, Rompala OJ, Verma K, Mortazavi MM, Benninger B, Loukas M, et al. Analysis of the uncinate processes of the cervical spine: an anatomical study. Journal of neurosurgery Spine. 2012;16(4):402-7. https://doi.org/10.31 71/2011.12.SPINE11541.

16. Kocabiyik N, Ercikti N, Tunali S. Morphometric analysis of the uncinate processes of the cervical vertebrae. Folia morphologica. 2017;76(3):440-5. https://doi.org/10.5603/FM.a2017.0010.

17. Sun JC, Yang HS, Shi JG, Yuan W, Xu XM, Shi GD, et al. Morphometric analysis of the uncinate process as a landmark for anterior controllable antedisplacement and fusion surgery: a study of radiologic anatomy. World neurosurgery. 2018;113:e101-7. https://doi.org/10.1016/j.wneu.2018.01.181.

18. Chen C, Ruan D, Wu C, Wu W, Sun P, Zhang Y, et al. CT morphometric analysis to determine the anatomical basis for the use of transpedicular 
screws during reconstruction and fixations of anterior cervical vertebrae. PLoS One. 2013;8(12):e81159. https://doi.org/10.1371/journal.pone.0081159.

19. Doherty BJ, Heggeness MH. The quantitative anatomy of the atlas. Spine. 1994;19(22):2497-500. https://doi.org/10.1097/00007632-199411001-00001.

20. Panjabi MM, Duranceau J, Goel V, Oxland T, Takata K. Cervical human vertebrae. Quantitative three-dimensional anatomy of the middle and lower regions. Spine. 1991;16(8):861-9. https://doi.org/10.1097/00007632-1991 08000-00001.

21. Doherty BJ, Heggeness MH. Quantitative anatomy of the second cervical vertebra. Spine. 1995;20(5):513-7. https://doi.org/10.1097/00007632-199503 010-00002.

\section{Publisher's Note}

Springer Nature remains neutral with regard to jurisdictional claims in published maps and institutional affiliations.

Ready to submit your research? Choose BMC and benefit from:

- fast, convenient online submission

- thorough peer review by experienced researchers in your field

- rapid publication on acceptance

- support for research data, including large and complex data types

- gold Open Access which fosters wider collaboration and increased citations

- maximum visibility for your research: over $100 \mathrm{M}$ website views per year

At BMC, research is always in progress.

Learn more biomedcentral.com/submissions 